\title{
Research on the mechanism of micro film advertising communication based on complex network
}

\author{
Lin Wang \\ LinYi University China \\ wanglin@lyu.edu.cn
}

\begin{abstract}
Key words: Micro film advertising, advertising communication, complex network, communication model

Abstract. Micro film advertising is a kind of propaganda means which is used to transmit information to the public for a specific need. The spread of the main micro film advertising is the initiator of brand advertising, which is mainly spread through two ways: one is the use of video sites, social networking sites, and ultimately the formation of fission spread; another by micro-blog and other communication means, and the same complex network information communication mode. In this paper, the propagation mechanism of micro film advertising on micro-blog and other new media is studied, and an improved SIR model is proposed.
\end{abstract}

\section{Introduction}

Due to the differences in the micro film advertising communication platform, so the video site and micro-blog platform as a node to the user to spread the way is also different. Micro film advertising through the interaction and feedback with different platforms and users, the advertisement information dissemination scope and depth has increased, and the process of communication mechanism respectively meet the fission spread and complex network of information dissemination.

\section{fission propagation mechanism}

The first mode of transmission of micro film advertising with the spread of fission, the micro film advertising and video sites, social networking sites such as the formation of networks, formed a reputation spread, in which the audience, video sites, social networking sites such as the spread of the source, forming a combination of multimodal communication and in the network communication.

In the process of transmission, the video information and the related comments or other information can be two-way flow. Because of the two-way flow of information, the advertising creators can change the way of communication and even the content of communication according to the feedback of the audience. Micro film advertising can help video website platform for the spread of fission, expanding the network level, and according to the node level feedback adjust or change the product advertising content and related marketing strategies etc..

\section{propagation mechanism based on complex networks}

Complex network is a large-scale network with complex topology and dynamic behavior, which is composed of a large number of nodes connected by edges. The basic research object of propagation dynamics is the relationship between the properties of the dynamical model on different networks and the static statistical properties of the network. Here, we introduce the SIR model to study the micro film advertising, in which $\mathrm{S}$ is the target audience, I for the spread of the 
crowd, $\mathrm{R}$ for viewing after the spread of the group. In actual communication, the target audience only through the dissemination of the individual can be infected, if each individual communication with a node represents a complex network, a part of the target audience for their formation of infected individuals, "two media" to the other audience, in the continuous forwarding process, the network is expanding constantly finally, the formation of the complex network; when micro-blog is forwarded to a node, but the node is not forwarded after watching the audience, micro film advertising information dissemination stop; visible, micro-blog forward more the number of nodes of the complex network more and more, spread effect of micro film advertising is more obvious.

The earliest spread of the network advertisement mainly through micro-blog, micro-blog continuously transmitted through the mouth, and the target audience has been "infected", which makes the product advertising has achieved great success. Visible, micro film advertising communication process and complex networks in the classic SIR propagation model.

\section{Propagation model of micro film advertising based on complex network}

Through the Internet to increase the number of people watching the micro film advertising A, every person who saw the micro film advertising can make a $\mathrm{s}(\mathrm{t})$ who did not see the micro film advertising into a micro film advertising. Because $I$ have seen the number of micro film advertising $\mathrm{N}_{\mathrm{i}}(\mathrm{t})$, so every time a total of aNs $(\mathrm{t}) * \mathrm{i}(\mathrm{t})$ who watched the micro film advertising people, namely $\mathrm{A}=\mathrm{aNs}(\mathrm{t})^{*} \mathrm{i}(\mathrm{t})$ (the average number of a for each read micro film advertising per hour in effective contact $\mathrm{N}$ is the total number of regions, in the micro film advertising period $\mathrm{i}(\mathrm{t})$ to see the micro film advertising in the total number of people in the proportion of $s(t)$ is not seen the micro film advertising accounted for the total number of people in the proportion).

Through the network information platform for the dissemination of new micro film advertising seen the number of $B$, each micro film advertising information platform can make the gem bs(t) has not seen the micro film advertising people read into the micro film advertising people. Because the total number of micro film advertising information platform for $\mathrm{M}$, so every time a total of bMs ( $\mathrm{t}$ ) who watched the micro film advertising people, namely $B=b M s(t)$ ( $b$ for each release of micro film advertising network information publishing platform average number per hour, effective browsing $\mathrm{M}$ for communication the total number of micro film advertising information platform).

\section{Propagation model}

Available from the above discussion, there is the propagation model as: $N \frac{\mathrm{d} i}{\mathrm{~d} t}=A+B$.

In this model, there is $\begin{aligned} & A=a N s(t) i(t) \\ & B=b M s(t)\end{aligned}$ and $\left\{\begin{array}{c}i(t)+s(t)=1 \\ i(0)=i_{0}\end{array}\right.$.

Therefore, the model can be expressed as:

$$
N \frac{\mathrm{d} i}{\mathrm{~d} t}=a N \cdot i(1-i)+b M(1-i)
$$

\section{Model solution}

Model can be transformed as: $\frac{\mathrm{di}}{a N \cdot i(1-i)+b M(1-i)}=\frac{d t}{N}$

$\int_{i_{0}}^{i} \frac{\mathrm{di}}{a N \cdot i(1-i)+b M(1-i)}=\frac{1}{N} \int_{0}^{t} d t$

Check the integral table and simplify it, there is: 


$$
i(t)=1-\frac{1}{\frac{a N i_{0}+b M}{(a N+b M)\left(1-i_{0}\right)} \mathrm{e}^{-\frac{b M+a N}{N} t}+\frac{a N}{a N+b M}}
$$

\section{Model analyze}

In the model as $i(t)=1-\frac{1}{\frac{a N i_{0}+b M}{(a N+b M)\left(1-i_{0}\right)} \mathrm{e}^{-\frac{b M+a N}{N} t}+\frac{a N}{a N+b M}}$, you can get $\mathrm{M}<<\mathrm{N}$, in the

extreme case:

(1)When the total number of micro film advertising in the region during the study of the micro film advertising in the total number of people in the proportion of 0 , that is, when $i_{0}=0$, $\lim _{t \rightarrow \infty} i(t)=0$

(2)When the total number of micro film advertising in the region during the period of micro film advertising in the total number of people in the proportion of 1 , that is, when $i=1$, $\lim _{t \rightarrow \infty} i(t)=1$

The results of this model are proved to be feasible by the results of (1) and (2).

\section{Conclusion}

The marketing effect of micro film advertising mainly depends on the unique communication mechanism of micro film advertising. In the analysis of the transmission mechanism, we believe that the micro film advertising spread mainly through two ways: on the one hand, through the spread of large video sites, social networking platforms, through the website of the audience - more audience this level fission spread clear, causing the attention of target audience and emotional resonance. On the other hand, spread through micro-blog, micro-blog users forwarding - part of the audience - users turn - more audience, compared to the spread of fission, the more nodes, two times greater impact, which is consistent with the propagation mechanism of complex networks, namely susceptible individuals through a node to a certain percentage of infection the target audience, the target audience continues to expand. Of course, there are many imperfect aspects of the SIR model, need to be made up in the future.

In addition, the micro film advertising of the two kinds of dissemination way have their advantages and disadvantages, the defect is due to the video in many video sites, only the top is more popular by the consumers, so for some micro film advertising, if the hot degree is low, may lose the potential target consumers. The latter is spread through the defect of micro-blog, when the immune population will gradually expand when to stop the spread of, if a micro film advertising target group is not clear, they may lose some of the micro film advertising interest, and ultimately the formation of immune and stop the spread, to reach the purpose of communication. So, I suggest, should be two modes of organic combination, to maximize the spread to video website communication as the basis, speed up the spread with micro-blog's forwarding function, caused by the appropriate heat and participation, so that consumers can get the corresponding information in browsing video website. In addition, by browsing the video site of the micro film advertising, in a timely manner to the corresponding viewing commentary micro-blog spread, making the micro film advertising can get long-term attention and dissemination efforts. 


\section{References}

[1]Exactly solvable small-world network. Dorogovtsev SN,Mendes JFF. Europhysics Letters . 2000 [2]Strong effects of weak interactions in ecological communities. Berlow E L. Nature . 1999

[3]Topological properties and transition features generated by a new hybrid preferential model. Fang J Q,Liang Y. Chinese Physics Letter . 2005

[4]Mathematical Biology. Murray JD. . 1993

[5]Measuring preferential attachment in evolving networks. Jeong H,Neda Z,Barabasi A L. Europhysics Letters . 2003

[6]Self-organized network evolution coupled to extremal dynamics. G. Caldarelli,A. Capocci,D. Garlaschelli. Nature Physics . 2007

[7]Generalized local-world models for weighted networks. Pan Zaofeng,Li Xiang,Wang Xiaofang. Physical Review E Statistical Nonlinear and Soft Matter Physics . 2006

[8]Multiple scales in small-world graphs. KASTURIRANGAN R. . 1999

[9] S.Valverde,,R.Ferrer i Cancho,,R.V.Sol啨. Europhysics Letters . 2002 